\title{
El derecho de la infancia a ser investigada correctamente
}

\section{María José Bernuz Beneitez}

Universidad de Zaragoza. Facultad de Derecho. Departamento de Derecho Penal Filosofía del Derecho e Historia del Derecho mbernuz@unizar.es

ORCID: 0000-0001-7723-5172

\section{Resumen}

Este trabajo presenta algunas reflexiones sobre la posibilidad de que las distintas consideraciones éticas a tener en cuenta cuando se realiza investigación con niños y niñas no se perciban como simples recomendaciones, sino que puedan conformarse como un derecho que exige el cumplimiento de algunas obligaciones al científico social que investiga en ese contexto. Para ello se analizan algunas especialidades a tener en cuenta en este tipo de investigación, como son la disimetría y las relaciones de poder entre el investigador y el investigado; la revisión de cuestiones clásicas, como el consentimiento informado o la confidencialidad, o los condicionamientos que marcan la participación igualitaria y significativa del menor en la investigación.

Palabras clave: derechos de la infancia; ética de la investigación en ciencias sociales; derecho a participar

\section{Abstract. The right of children to be researched properly}

This paper presents some reflections on the possibility that the different ethical considerations to be taken into account when conducting research with children are perceived as a right and not just simple recommendations. To this end, we analyse some specific aspects of this type of research, such as dissymmetry and the power relations between the researcher and the researched, the revision of classical issues such as informed consent or confidentiality, or the conditions that mark the equal and meaningful participation of children in research.

Keywords: children's rights; social sciences research ethics; participatory rights 


\section{Sumario}

\begin{tabular}{|c|c|}
\hline 1. Introducción & 5. Entre la participación efectiva \\
\hline 2. El derecho a ser investigado entre & \\
\hline los derechos de la infancia & \multirow{2}{*}{$\begin{array}{l}\text { 6. Conclusiones: sobre las } \\
\text { consideraciones éticas y el respeto } \\
\text { de los derechos de las personas } \\
\text { investigadas }\end{array}$} \\
\hline $\begin{array}{l}\text { 3. La cuestión de la disimetría entre } \\
\text { investigador e investigado }\end{array}$ & \\
\hline $\begin{array}{l}\text { 4. Cuestiones clásicas (pero revisadas) } \\
\text { en la investigación con niños: } \\
\text { la confidencialidad (y el secreto) } \\
\text { y el consentimiento informado }\end{array}$ & Referencias bibliográficas \\
\hline
\end{tabular}

\section{Introducción}

Unos años atrás hice una investigación empírica sobre la implementación de la ley reguladora de la justicia de menores vigente en aquel momento, la LO 4/92. Entendía que si investigaba sobre la puesta en marcha de un nuevo modelo de justicia de menores era preciso escuchar la voz de sus destinatarios, esto es, los operadores que se encargan de dar vida a la normativa, pero también los jóvenes y adolescentes que cometen delitos y deben ser enjuiciados bajo esa legislación. En ese marco y con el objetivo de conocer cómo habían percibido el paso por las instituciones judiciales de menores, hice cinco entrevistas semiestructuradas a cuatro chicos y una chica ${ }^{1}$ que habían cumplido con medidas de libertad vigilada, servicios en beneficio de la comunidad, reparación a la víctima, o que estaban cumpliendo en ese momento con una medida de internamiento en régimen semiabierto. El acceso a los menores se produjo a través de los educadores que habían trabajado con ellos (que hicieron de porteros o gatekeepers) y que se encargaron de gestionar el día y el lugar de la entrevista. Las dificultades legales para permitir el acceso a menores de dieciocho años hicieron que se me propusiera la posibilidad de realizar las entrevistas con quienes ya habían llegado a su mayoría de edad y, por ello, bastó con su consentimiento.

Las entrevistas se desarrollaron según el plan previsto, el ambiente fue cordial y distendido, pero siempre tuve la impresión de que algo no encajaba. No sabía si la sensación tenía que ver con la inevitable forma de acceder a los menores, que cuenta con el sesgo de que ellos son "preseleccionados» por los educadores, o si se debía a que creía estar ante un público cautivo, cuyo consentimiento no era totalmente libre por estar "de alguna manera» comprometidos con la institución. No sabía si el problema estaba en mi (desin) formación para entrevistarme con adolescentes y jóvenes. Quizás, un prejuicio etario y adultocéntrico me hacía pensar que la información que me ofrecían los jóvenes entrevistados no se ajustaba a «la» realidad, sino a una realidad idealizada y adaptada a lo que pensaban que los profesionales (que por supuesto

1. Proporción ajustada a la distribución por sexos de la delincuencia juvenil en España. 
no estaban presentes) querían escuchar cuando leyeran el texto en el que se volcarían sus opiniones. También pensé que mi posición adulta permitiría una interpretación exclusivamente externa (emic) sin atisbos de que pudiera ser mínimamente empática (etic).

De esa limitada experiencia, quedó el interés por trabajar más en profundidad sobre las especificidades que debe tener (o no) la investigación en ciencias sociales que implica a niños y adolescentes. No solo por las precauciones que debe tener el investigador al planificar y realizar su trabajo, sino también por las implicaciones que puede tener la propia investigación para el investigado menor de edad. Unas y otras deberían, quizás, conformar un derecho de niños y niñas a ser investigados adecuadamente, lo que debería traducirse en unas obligaciones a cumplir por los investigadores ${ }^{2}$.

Este hipotético derecho del niño a que se le investigue de forma apropiada nos exige confrontar temas que en nuestro país se han estudiado por separado sin que hayan confluido en una reflexión conjunta. Así, de un lado, es abultada la bibliografía sobre la transformación de la concepción (que no la realidad) de la infancia alentada por la Convención de Naciones Unidas sobre los Derechos de los Niños (en adelante, CDN) y la especificidad de los derechos de protección, promoción y participación que propone. También empiezan a ser abundantes las investigaciones empíricas en torno a las dificultades que presenta la efectiva implementación de los derechos de la infancia o a la correcta adaptación de las instituciones para que niños y niñas puedan disfrutar de sus derechos en igualdad de condiciones respecto a los adultos. De otro lado, existe una considerable bibliografía sobre las cuestiones éticas a tener en cuenta en el desarrollo de la investigación en ciencias sociales, sea por la relación entre el investigador y el investigado (que se da más intensamente en estudios cualitativos y/o etnográficos), por las consecuencias que la investigación puede tener en el sujeto investigado o por el tratamiento de la información confidencial, ilegal o de otra índole, que pueda sacar a la luz la propia investigación.

$Y$, aunque la relación entre derechos y ética parece clara porque esta última tiene que ver con la dignidad de las personas y entendemos que la dignidad es indisociable del respeto de los derechos, lo cierto es que Bell (2008: 7-8) ha reconocido que esa vinculación fuerte se ha desconocido o se ha ignorado, y la comunidad científica parece preferir hablar de principios y de guidelines en lugar de derechos de las personas en la investigación. Aunque se trata de un tema abordado exhaustivamente en la literatura anglosajona, también algunos científicos sociales españoles evidencian las exigencias éticas y metodológicas a tener en cuenta en la investigación con niños y niñas ${ }^{3}$. En ese marco, parecía interesante revisar algunas de las implicaciones que tiene considerar los

2. El título que dan Beazley et al. (2004) a su trabajo inspira el que se presenta ahora.

3. Véase, entre otros, Rodríguez Pascual (2007) sobre algunos retos metodológicos en la investigación con niños y adolescentes, así como Gaitán (2006) y Gaitán y Liebel (2011) para una vinculación entre los derechos de participación de la infancia y su integración en investigaciones sociales sobre infancia y adolescencia. 
derechos de participación del niño en la investigación en ciencias sociales. Más precisamente, evidenciar que son esos derechos de participación los que exigen que las investigaciones sobre temas que les incumben cuenten, siempre que sea posible, con sus opiniones y visiones. O que es preciso que las cuestiones de deontología de la investigación se revisen y se adapten cuando esta cuenta con niños y niñas como sujetos de la investigación. Por ello, el objetivo esencial de este trabajo no es tanto el de ofrecer una guía, sino más bien el de alentar y continuar una discusión colectiva sobre las precauciones éticas y metodológicas a considerar cuando se hace investigación social con niños y adolescentes. Sobre todo porque no tenerlas en cuenta puede suponer una violación de sus derechos.

\section{El derecho a ser investigado entre los derechos de la infancia}

Es cierto que la CDN no reconoce el derecho a ser investigado correctamente como tal, pero, como indican Beazley et al. (2004: 370), el derecho del niño a ser investigado adecuadamente tiene que ver con otros derechos reconocidos efectivamente en la CDN, como son el derecho del niño a ser escuchado, a dar su opinión y a que esta se tenga en cuenta en función de su madurez (art. 12), el derecho a la libertad de expresión por el medio que el propio menor elija y a buscar información por todos los medios posibles (art. 13), el derecho a ser protegido contra cualquier forma de explotación que no haya sido reconocida en otros apartados de la propia Convención (art. 36) y el derecho a que se desarrollen los más altos estándares posibles de protección, sobre todo de salud y seguridad en el trabajo con niños (art. 3.3). En otras palabras, se podría decir que la investigación social, descuidada metodológica y éticamente, puede atentar contra varios derechos de la infancia reconocidos en la normativa internacional y nacional. Como establecen los autores, se trata de derechos y principios que, cuando se llevan al ámbito de la investigación con niños, significa que estos puedan participar «en la investigación, que se utilicen métodos que les permita expresar sus opiniones, puntos de vista y experiencias fácilmente», pero también, "que se les proteja del daño que les puede suponer involucrarse en la investigación, que esté dirigida por investigadores que usen métodos y análisis científicos de calidad» (Beazley et al., 2004: 370). Son, todos ellos, derechos que tienen que ver con la promoción de la participación del niño en decisiones que puedan afectar a sus vidas (individualmente o como colectivo). De esta manera, «las voces de los menores se han convertido en el símbolo del compromiso del moderno estado de bienestar con los valores de libertad, democracia y cuidado" (James, 2007: 261).

En todo caso, junto a los esfuerzos de la CDN por transformar normativamente la forma de entender la infancia, lo cierto es que también ha habido una crítica doctrinal a las teorías existentes sobre la infancia ${ }^{4}$. Entre

4. Se puede ver una presentación muy clara de los enfoques estructural, construccionista y relacional de la nueva sociología de la infancia en Gaitán (2006). 
ellas, se han ido abandonando progresivamente las teorías de la socialización que asumían que el niño era un ente vacío en el que volcar ideas sociales sin ningún tipo de comentario o aportación por parte del menor, o la psicología del desarrollo, que asume un proceso lineal de crecimiento y madurez del niño que va de la simplicidad a la complejidad y desde la irracionalidad hasta la racionalidad. Crítico con esos planteamientos, Kirk (2007: 1252) asegura que «se conceptualizaba a los menores como inmaduros, incompetentes y como objetos conformes y pasivos en un proceso unidireccional de socialización». También Rodríguez Pascual (2006: 66) asegura que «nuestras suposiciones sobre la vida social de los menores subestimaban sistemáticamente la capacidad de éstos para interpretar e influir sobre las situaciones sociales». Esos desarrollos teóricos, junto con el proteccionismo institucional hacia grupos vulnerables, podían convertir a niños y niñas en «sujetos inherentemente vulnerables o reforzar su vulnerabilidad estructural en la sociedad, comprometiendo su derecho a participar legítimamente en la investigación que le afecte» (Chakraborty et al., 2012: 542-543). Y todo ello se realizaba, claro está, por el bien del menor y con el objetivo de protegerle. Quizás lo que tendríamos que comprender es que los niños pueden ser vulnerables, pero al mismo tiempo competentes para participar en una toma de decisiones y, por supuesto, en una investigación. Si bien es cierto que, como indica Komulainen (2007: 26), en la práctica, la decisión sobre si prima la vulnerabilidad o la competencia, parece que la toman siempre los adultos.

Así pues, tanto los nuevos desarrollos sobre la infancia como el marco normativo que aporta la $\mathrm{CDN}$ han sido esenciales para promover un progresivo cambio en la forma de entender la infancia y considerar a niños y niñas como sujetos de derechos con una voz propia que debe ser considerada. Para Fernandes (2016: 763), se trata de perspectivas teóricas que consideran al menor como un actor social «con una acción socialmente relevante, sujeto de derechos y un ser competente en sus mundos de pertenencia en el que estructura relaciones sociales constitutivas de órdenes sociales infantiles».

En todo caso, la propia concepción de la infancia que defiende la CDN como sujeto de derecho al que le corresponden derechos de protección, de promoción y (novedad) de participación ha fomentado que también la ciencia sea cada vez más consciente de que las investigaciones sobre infancia deben contar con la opinión de los adultos sobre los niños, pero también con la de los propios menores de edad. Ellos tienen experiencias vitales e intereses distintos de los de los adultos, deben ser actores sociales participativos y, por ello, deben ser escuchados en cuestiones de su interés, porque la versión que dan las personas mayores sobre su vida y sus experiencias puede no ser la correcta. No hacerlo así, esto es, realizar solo investigación social sobre niños, sin promover investigación con ellos, estaría contribuyendo a lo que Santos y Meneses llaman epistemicidio del conocimiento, por desconocer aquello que no sea pensamiento dominante e ignorar la riqueza que aporta la heterogeneidad etaria (en Fernandes, 2016: 762). Fernandes también alerta de que se puede dar un eticidio del conocimiento cuando los sujetos no estén informados de que 
se les está investigando o sean informados pero la investigación sea totalmente diseñada por adultos (Fernandes, 2016: 762-763). Algo que sería indicativo de la total falta de respeto y consideración de las personas mayores hacia los niños y niñas investigados, que no es sino el reflejo de la falta de consideración que el adulto muestra cotidianamente hacia sus opiniones y puntos de vista (Morrow, 2008: 51), o señal de que el investigador no los sigue considerando como sujetos, sino como objetos de su investigación sobre los que disponer libremente. Se trata de una actitud que atenta contra uno de los derechos esenciales del niño, que es el de ser tratado con dignidad.

Pese a ello, no podemos obviar que es algo aceptado por la doctrina que los derechos de la infancia surgen en un proceso de especificación, esencialmente porque se entiende que la infancia conforma un colectivo social con unas necesidades especiales. Ello significa que las instituciones (también las científicas) deberán hacer las adaptaciones necesarias para que niños y niñas puedan disfrutar de sus derechos en las mismas condiciones en que lo harían los adultos. Esas especificidades deben considerarse igualmente cuando se realiza investigación social con niños. Y, aunque es cierto que en la investigación en ciencias sociales hay cuestiones éticas y metodológicas a considerar al margen de que el sujeto investigado sea niño o adulto, otras sí que son específicas, porque, como aseguran Thomas y O'Kane (1998: 337), los niños tienen una forma distinta de ver el mundo y de comunicarlo y porque experimentan las relaciones de poder de manera diferente.

Quizás porque en el marco de la investigación social se producen indudablemente relaciones de poder y dominación, la protección frente al abuso o a la manipulación deben verificarse con más rigor cuando se realiza investigación con niños. De hecho, vemos que las instituciones que permiten el acceso del investigador a los niños tienden a la sobreprotección, porque asumen que estos son menos capaces de comprender el alcance de sus decisiones y entienden que estas les pueden hacer más vulnerables. También analizaremos cómo la confidencialidad de la información revelada en la investigación es constantemente cuestionada por los adultos responsables, que creen tener «derecho» a conocer lo que han contado los niños al investigador, quizás sin ser conscientes de que también los adultos responsables de los niños ejercen una relación de poder sobre estos, que no siempre es beneficiosa para ellos.

\section{La cuestión de la disimetría entre investigador e investigado}

Una de las cuestiones más interesantes para reflexionar en investigación en ciencias sociales es la que tiene que ver con la posición que ocupa el investigador cuando realiza su trabajo y la influencia que este puede tener en el sujeto investigado, en el propio resultado de la investigación y en la presentación de resultados (Morrow, 2008: 52). Es cierto que algunas diferencias de posición las puede marcar el tipo de investigación que se realice. Así, en la cuantitativa, el investigador se suele presentar como el experto sin paliativos. Es él quien conoce el método, determina las variables, diseña el cuestionario que es respon- 
dido de forma casi mecánica por el investigado y conoce las herramientas de análisis y categorización de la información. En la investigación cualitativa y/o etnográfica, el investigado es el experto en el tema objeto de investigación y el investigador el que trata de adaptarse a sus tiempos, registros y requerimientos. Sin embargo, tanto en la investigación cuantitativa como en la cualitativa, lo cierto es que es difícil para el sujeto investigado oponerse a la autoridad del investigador y resistirse a la "mística» del propio estudio científico. Son aspectos que marcan una distancia difícil de salvar en todo caso, y más cuando el investigado es menor de edad.

En cualquier fase de la investigación parece evidente que, cuando el investigador es un adulto y el investigado es un niño, la disimetría de poder entre uno y otro es doblemente palmaria y evidente, por el propio estatus del investigado frente al del investigador y por ser un niño frente a un adulto. Rodríguez Pascual (2006: 70) señala que «dicha asimetría radica de forma permanente en posiciones o estatus que ni el investigador ni el niño pueden evitar». Soares propone pensar la ética "como un recurso para lograr el equilibrio entre el investigador y los niños investigados» (en Pereira y Barros, 2011: 152). Y es cierto que la reflexión sobre la disimetría entre investigador e investigado tiene que ver con cuestiones de deontología y metodología, porque esa situación de desequilibrio puede condicionar y perturbar la participación del menor en la investigación, que debe ser, en todo caso y momento, libre y voluntaria. Como se pregunta Kirk (2007: 1252): «chasta qué punto son libres los menores para participar o negarse a seguir participando en la investigación?». Y ello, insisto, tanto por su condición de investigado como por su condición de menor frente al investigador adulto, o frente a los adultos responsables de él, que consienten o no su participación. Es igualmente claro que la disimetría puede condicionar también la respuesta más o menos espontánea del menor. Así, Fernandes (2016: 770) destaca que, teniendo en cuenta la diferencia de poder entre el niño investigado y el investigador adulto, debemos preguntarnos hasta qué punto podemos estar seguros de que los niños «dan sus propias opiniones o comparten experiencias con los investigadores adultos sin sentirse forzados e influenciados en la forma en que producen (o no) con autoría sus representaciones». Spyrou (2011: 154, 152,153) concluye afirmando que las relaciones de poder entre investigador e investigado «mediatizan la producción de investigación", pueden condicionar lo que se cuenta y su relación con la verdad, al mismo tiempo que también el método y los mecanismos de acceso utilizados para investigar pueden condicionar el acceso al menor y su disponibilidad o su capacidad para comunicar de manera significativa para él y para el investigador.

Parece claro que, al margen de cuál sea la metodología por la que se opte, la disimetría más evidente entre investigador e investigado se va a producir en el momento de informar y dar cuenta de los resultados del estudio. El investigador será quien finalmente redacte el informe o escriba el artículo y, en consecuencia, pueda decidir qué iluminar, integrándolo en el texto, y qué es lo que queda fuera porque escapa a los objetivos previstos en la investigación. Algo que se ha denominado "autoría autoritativa» y que se produce en cualqui- 
er investigación (James, 2007: 265), pero que se intensifica cuando se trata de una investigación con niños, por la inexistencia de vías de queja para oponerse a lo dicho en la investigación o porque en ocasiones no tendrán acceso a los resultados de la misma5.

Desde la perspectiva del investigador, la disimetría también se traduce en la existencia de barreras relacionadas con su acceso al conocimiento. Pereira y Barros (2002: 153) se preguntan, "¿hasta qué punto es posible alcanzar una visión del mundo infantil?». Quizás sea preciso asumir esta incapacidad y asimilar que «un adulto nunca puede ser "nativo" en las palabras de los niños, no solo por la diferencia de edad, sino por el estatus privilegiado que lo identifica como adulto y que el investigador, como adulto, lleva consigo" (Spyrou, 2011: 156). Como asegura Ignatieff (2000: 108), los niños deben ser tratados «como agentes morales en sí mismos, con intenciones, objetivos y visiones del mundo que no deberíamos presumir idénticos al nuestro». Punch (2002: 324-325) considera fundamental no asumir que entendemos a los niños y comprendemos sus visiones porque también lo fuimos ${ }^{6}$ — dada nuestra capacidad para desaprenderlo rápidamente-, ni partir de que el conocimiento adulto es superior al de los niños. Como aseguran Gallagher y Gallagher (2008), igual hay que apostar por una investigación que se asuma como inmadura y en constante proceso de reconstrucción y por un investigador que se rechace como experto y asuma su incapacidad para comprender el mundo de niños y jóvenes. De otra manera, como asegura Geertz (1988, en James, 2007: 263), estaríamos haciendo etnografía ventrílocua cuando reclamamos no hablar de otra forma de vida, sino desde dentro de ella, y nos presentamos «como capaces de ver el mundo desde la perspectiva del niño como una nueva forma de verdad».

En esa línea, también es clara la disimetría cuando valoramos como "correcto» o no, como ajustado o no a la realidad, lo que dice el niño. Así, en ocasiones, se asegura que la investigación hecha con menores muestra «una representación auténtica de las voces de los niños», cuando, en realidad, lo que hace el investigador es traducir e interpretar sus voces y llevarlas a un registro más «adecuado» (James, 2007: 263, 267). El adulto da sentido a aquello que el menor «ignora, omite o no responde» (Spyrou, 2011: 157). Parece que somos «incapaces de reconocer lo que hay de no-normativo y no-domesticado en sus voces [...] el investigador impone sus propios sentidos en la información que

5. Harcourt y Quennerstedt (2014: 5) anteponen el riesgo de coerción de los niños por parte de los adultos para participar o no en una investigación, así como el posible conflicto que se puede producir entre los intereses del menor y los de sus padres o responsables. Morrow (2008: 58) asegura que los niños, en tanto grupo social vulnerable, no pueden cuestionar las formas en las que se presentan los datos de las investigaciones sobre ellos.

6. Asegura Rodríguez Pascual (2006: 84) que «es difícil para el científico social escapar al recuerdo (y pre-noción) de su propia infancia, lo que convierte la interpretación de la vida social del niño en una (re)interpretación de la infancia tal y como ha sido experimentada por el investigador». Por ello recomienda una prudente distancia que nos mantenga alerta sobre nuestra forma de interpretar la realidad conforme a nuestro conocimiento previo (Rodríguez Pascual, 2007: 78). 
obtiene de los niños [...] lo que los niños dicen puede ser interpretado como que significa lo que el investigador entiende, más que lo que el niño quiere decir» (Spyrou, 2011: 158). A veces intentamos encajar sus intenciones e ideas con situaciones definidas por los adultos (Komulainen, 2007: 25). Así, puede ocurrir, como advierte Fernandes (2016: 774), que «la semántica del adulto se sobreponga a la semántica del niño, todo aquello que el niño dice puede ser utilizado al servicio de lo que el adulto entiende que es lo que el niño quiso decir».

Otras veces se considera, en sentido contrario, que el conocimiento que transmiten los menores no es auténtico o verdadero. Algo que puede tener que ver con el hecho de que los adultos no tienen competencias suficientes o adecuadas para interpretar lo que dicen los niños o tienden a usar lo que dicen simplemente para confirmar prejuicios del investigador (James, 2007: 262) ${ }^{7}$. Como aseguran Gaitán y Liebel (2011: 171), si la comunidad científica excluye a los niños por entender que «no saben», «los investigadores adultos que tratan seriamente de "dar voz a los niños" en la investigación corren el riesgo de violar lo que se entiende por buena conducta científica».

Se podría concluir que, si el investigador considera importante la participación del menor y la autenticidad de su respuesta, debería cuidar que las condiciones permitan equilibrar la disimetría entre investigador e investigado. Es evidente que cuanto más adaptados sean el espacio y la metodología, más real será la conformidad informada que encontremos por parte del menor para participar en la investigación, más capaz será el menor de hacerlo de manera significativa y de mayor calidad será su respuesta. Como aseguran Thomas y O’Kane (1998: 341), «factores como cuándo y dónde se realiza la entrevista, quién está presente, a quién se informará, tendrán un efecto probable sobre lo que cuente el menor». Igualmente, si el investigador aspira a ofrecer confianza al sujeto investigado para que pueda participar libremente y hacerlo con sinceridad y honestidad, tiene que tener en cuenta otros factores que pueden estar condicionando la respuesta, como pueden ser, entre otros, el estatus, el género, la discapacidad, la etnia o la cultura. Pero, sobre todo, deberá atender a su edad, porque, como aseguran Beazley et al. (2009: 368), «en la infancia, las diferencias de edad pesan más que el género, la etnia, la religión u otros factores de discriminación». Algo que exige hablar de «las voces de los niños» y mostrar una consideración individual, no solo grupal, de sus opiniones (James, 2007: 262).

\section{Cuestiones clásicas (pero revisadas) en la investigación con niños: la confidencialidad (y el secreto) y el consentimiento informado}

Cuando hablamos de cuestiones éticas en la investigación con niños, una pregunta esencial es la siguiente: «¿Cambian estas según hagamos investigación con niños o con adultos?». Morrow (2008: 51) considera que deben ser iguales, al

7. Otros autores consideran que la tendencia a incorporar y hacer participar al menor en la investigación social es perversa, porque aspira a comprender mejor a niños y jóvenes con el objetivo de regularlos mejor (Gallagher y Gallagher, 2008). 
margen de quién sea el sujeto de estudio, la honestidad en el proceso de recolección, análisis, interpretación y difusión de los datos, así como la protección de los sujetos de la investigación. Más precisa y más ampliamente, Kirk (2007: 1258 ) indica algunas cuestiones que igualan, aunque con matices, la investigación con niños a la realizada con adultos. Así, asegura que deberían ser similares el respeto y la consideración a la individualidad de cada una de las personas investigadas, la necesidad de valorar y tomar en consideración las diferencias que puedan condicionar el resultado de la investigación (género, etnia, cultura, etc.) y la consiguiente exigencia de utilizar métodos adaptados a esas diferencias (la dificultad de la infancia para hablar directamente con adultos que no conocen, con menor capacidad de concentración cuando se trata de niños de corta edad), la necesidad de utilizar un lenguaje adaptado a la población a la que se dirige la investigación, tener en cuenta el contexto de investigación valorando si el sujeto prefiere que sea en su espacio o en otro ajeno, que el investigador no imponga su punto de vista (más frecuente cuando el investigado es un niño), que sea claro en la explicación de herramientas y preguntas de investigación, que solo investigue cuando y sobre lo que tenga consentimiento informado, que pueda asegurar la confidencialidad y, en todo caso, que evite los posibles daños que pueda causar la participación en la investigación.

Sin embargo, destaca Kirk (2007) que también hay algunas diferencias a tener en cuenta en la investigación con niños. Así, hay que asumir que la forma infantil y juvenil de ver el mundo es diferente a la de los adultos, puesto que los jóvenes tienen un grado de desarrollo cognitivo acorde a su edad y madurez y muestran un nivel de competencia diferente para comunicar. Por ello el investigador se encuentra con una mayor dificultad para establecer una relación de investigación con niños y adolescentes (que tienden a mirar con recelo a los adultos ${ }^{8}$ ), y el acceso a los niños se produce casi siempre a través de unos adultos que pueden restringir y/o limitar el acceso del investigador. Como consecuencia, es preciso idear métodos que favorezcan una participación significativa tanto para el niño como para el investigador ${ }^{9}$.

Quizás destacamos estas cuestiones que tienen que ver con la relación del investigador con los menores investigados porque entendemos que el conocimiento y su generación no son tanto factuales como relacionales y dependen, en parte, de las relaciones que establecemos con quienes investigamos. También por ello muchas de estas cuestiones tienen que ver con la metodología de investigación, pero, como indica Punch (2002: 323), sobre todo están relacionadas con la deontología de la propia investigación. A continuación hacemos un apunte sobre dos cuestiones que adquieren tintes específicos cuando se investiga con niños: el consentimiento informado y la confidencialidad.

8. En ese sentido, es interesante la cuestión sobre cómo se debe presentar el investigador para evitar la confusión pero sin pretender el engaño. En unas ocasiones se opta por presentarse como un «invitado» (Christensen y Prout, 2002: 487).

9. Véanse algunos ejemplos de metodología cualitativa adaptada a la investigación con niños en Gaitán (2006: 249-255), así como en Rodríguez Pascual (2006). 


\subsection{El consentimiento informado}

Una de las cuestiones clásicas en la deontología de la investigación en ciencias sociales tiene que ver con el consentimiento del sujeto de investigación, que siempre debe ser previamente informado. Dicho aspecto es especial en el caso de un menor, dado que, para que él pueda consentir, previamente ha debido consentir un adulto responsable (que veremos en un apartado posterior).

Parece haber acuerdo en que algo fundamental en cualquier proceso investigador es que el sujeto investigado, debidamente informado sobre los aspectos básicos de la investigación (quién, cómo, por qué, para qué, etc.), pueda decidir libremente si participa o no en la investigación, lo haga en las cuestiones que considere oportunas ${ }^{10}$ y pueda declinar su consentimiento para participar en cualquier momento y por las razones que considere oportunas ${ }^{11}$. Y para lograr este consentimiento, que debe mantenerse constante a lo largo de la investigación, el investigador deberá adaptar su registro de comunicación al del menor, ser asertivo e insistir cada cierto tiempo o en cada fase de la investigación (cuando se trata de investigaciones prolongadas en el tiempo), indicándole las implicaciones de consentir y también la ausencia de consecuencias que tendrá el disentir o renunciar a seguir con la investigación.

Igualmente, parece claro que el investigador, al informar, tratará de equilibrar la protección de los intereses de los niños investigados con el desarrollo de la propia investigación (Christensen y Prout, 2002: 488), sobre todo porque es consciente de que, en ocasiones, si los niños hubieran conocido la finalidad de una grabación o una observación, quizás no hubieran consentido en participar en la misma. Y es que, como reconocen los autores, no siempre es fácil o posible calificar el consentimiento del menor como real (Beazley et al., 2004: 373). De hecho, Gallagher y Gallagher (2008) consideran que los jóvenes pueden entender la participación en la investigación como parte de un proceso de socialización, una conformidad a las normas adultas y una forma de dar valor a la cultura de los mayores sobre la suya propia.

Conscientes de estas dificultades, el consentimiento informado en investigaciones con menores requiere una serie de exigencias adicionales. Así, Graham et al. (2015: 338) consideran que el consentimiento debe ser siempre explícito, de modo que solo se podrá considerar válido si los niños han sido informados previamente y de forma adecuada a su capacidad de comprensión sobre los términos esenciales de la investigación, así como sobre los riesgos y beneficios de la participación y de la propia investigación. Debe ser un consentimiento libre y sin coerción (algo que requiere especial atención cuando se trata de niños más susceptibles de manipulación por parte de los adultos con

10. Es preciso reconocer que el derecho a participar también significa poder guardar silencio en las cuestiones que considere oportunas (Clarke y Moss en Christensen, 2004: 170).

11. Para Kirk (2007: 125) el consentimiento informado se apoya en tres aspectos: $a$ ) que los participantes sean informados de forma comprensible, $b$ ) que consientan voluntariamente y $c$ ) que sean competentes para dar su consentimiento. Powell y Smith (2009: 130) anteponen que el investigador sepa detectar signos de estrés del niño o su oposición a hablar de algún tema. 
los que está en relación) y debe poder renegociarse o rechazarse en cualquier momento de la investigación ${ }^{12}$. Más precisamente, Fernandes (2016: 166) recuerda que el consentimiento debe ser un proceso cambiante y «dependiente de la relación social de investigación que se va construyendo, así como de las interpretaciones que los niños hacen sobre la persona del investigador o la investigadora».

\subsection{El anonimato y la confidencialidad}

Otra de las cuestiones éticas más consolidadas en la investigación en ciencias sociales es la que tiene que ver con el anonimato y la confidencialidad. Son cuestiones que no se pueden confundir. Como dijimos en otra ocasión: «El anonimato significa que el investigador no pueda identificar la información con el informante [...] La confidencialidad tiene que ver con el compromiso por parte del investigador de no hacer pública la relación entre la información y el informante» (Bernuz et al., 2018). Esos principios, y básicamente el de la confidencialidad, deben imponerse y reforzarse en la investigación con niños si tenemos en cuenta su situación de especial vulnerabilidad y dependencia de los adultos o la importancia que conceden al secreto. Quizás por ello resulta curioso comprobar cómo los porteros que permiten el acceso a los niños y niñas investigados (padres, profesores, expertos sociales, etc.), en principio tendentes a velar por sus intereses, parecen comprender la necesidad del anonimato y exigen confidencialidad en la investigación, pero insisten en saber qué dicen los menores cuando están solos con el investigador y, sobre todo, qué cuentan sobre su vida diaria en la institución en la que se encuentran (escuela, hospital, centro de protección o de internamiento), intentan interpretar (en un sentido adulto) lo que los niños dicen o construyen un «sentido común» entre el investigador y el niño (Christensen y Prout, 2002: 486).

Se diría que, para ellos, el pacto de confidencialidad con el menor debe claudicar ante personas que velan por su bien. Algo que puede encubrir inseguridad y miedo a que emerjan cuestiones que les comprometan o evidenciar una idea de que los niños no deben o no pueden tener secretos para sus mayores, cuando, en realidad, como indica Christensen (2004: 171), el pacto de confidencialidad es algo realmente importante para los niños «cuyas relaciones y amistades se construyen a menudo en torno al compromiso a contar o guardar secretos, contar secretos a otros niños o contarlos a los adultos». Se trata de situaciones que, como indica la autora, colocan al investigador en una situación de compromiso porque debe mantener el pacto de confidencialidad con los niños, pero se ve presionado por los adultos responsables del menor, que pueden limitar su acceso a ellos y limitar el alcance del estudio.

12. En esa tarea de autocrítica del investigador este debe poder preguntarse si todos los niños son capaces de consentir, si es preciso el consentimiento de los padres, cómo asegurarse de que los niños estén bien informados, que su consentimiento sea libre y cómo respetar efectivamente su deseo de no participar en la investigación. 
Otra de las cuestiones que se pueden plantear cuando se hace investigación con niños, y que puede poner en cuestión el compromiso de confidencialidad, tiene que ver con el hecho de que el investigador, con su trabajo, conoce la vida del niño e interfiere en ella. La duda es qué tendría que hacer el investigador si, durante la investigación, descubriera una situación del menor que debería ser conocida por las autoridades para que decidieran si intervenir a través de los mecanismos de protección o judiciales adecuados (absentismo, abusos, explotación, negligencia, delincuencia, etc.). En este caso, la duda está en si es preferible mantener el compromiso de confidencialidad con el menor o romperlo para alentar su protección. Unos, como el National Children’s Bureau, apuestan por poner el caso en conocimiento de las autoridades. Se podría decir que, si somos consecuentes con la concepción del niño como sujeto de derecho, antes de tomar cualquier decisión, lo mejor es hablar con él, discutir la situación y ver con él la mejor manera de proceder (Graham et al., 2013: 77 s.).

\section{Entre la participación efectiva del menor y su protección}

Ya decíamos que la CDN incorpora, junto a los derechos de promoción y de protección de la infancia, los derechos de participación. Uno de los derechos de participación esenciales es el derecho del niño a ser escuchado en las cuestiones que le incumben y son importantes en su vida (art. 12). Y parece haber acuerdo en que el derecho a participar en investigaciones relacionadas con los menores y a que se escuche adecuadamente su opinión son importantes para el niño como individuo, porque le empodera y le responsabiliza (si está bien diseñada la investigación y no lo considera como simple generador de información), pero también porque puede tener repercusión en la infancia como colectivo. De hecho, Gaitán y Liebel (2011: 89) destacan lo siguiente:

[...] los niños deben tener la posibilidad de dar a conocer sus puntos de vista, muy especialmente cuando éstos se refieren a derechos que hasta el momento no les alcanzan a ellos, o a derechos que los niños evalúan y ponderan de manera diferente que las autoridades correspondientes o la sociedad adulta en general.

Por ello es importante que la participación del menor sea significativa. Es decir, que la propia investigación resulte interesante e importante para él y que este pueda comunicar y transmitir sus experiencias y percepciones de forma efectiva. En ese contexto, es preciso analizar la cuestión de quién (y en qué condiciones) puede participar, para vislumbrar la tensión adulta que se produce entre promover la participación y/o la protección de los niños y conocer qué entendemos por participación.

\subsection{Participación significativa versus adaptación metodológica}

Está claro que el menor tiene derecho a intervenir en aquellas decisiones que puedan ser importantes en su vida, y puede serlo el participar en una inves- 
tigación. Ahora bien, ello supone tanto intencionalidad (deseo consciente) como performatividad (hacer algo) (Gallagher y Gallagher, 2008) ${ }^{13}$. Y parece evidente que, para que esos dos factores confluyan, la participación debería ser significativa para el menor. Esto es, tiene que comprender el sentido de la investigación o su participación en ella debe resultarle estimulante de alguna manera. Pero además, previamente, se le debe haber empoderado (Gallagher y Gallagher, 2008). Y eso comúnmente se considera que es tarea del investigador, que deberá hacer un esfuerzo por adaptar formalmente los métodos a las capacidades del menor ${ }^{14} \mathrm{o}$, incluso, debe intentar integrarlo en el propio proceso investigador. Autores como Thomas y O'Kane (1998: 341-342) consideran que se puede hacer participar al menor de diferentes maneras y en distintos grados, sea acordando con él temas y cuestiones que puedan ser relevantes y de interés para su persona, utilizando técnicas de investigación participativa que den al niño una mayor sensación de control sobre el proceso investigador y que revaloricen lo que tiene que decir, o fomentando actividades con las que compartir puntos de vista e ideas, en las que él pueda redefinir cuestiones planteadas con anterioridad o el investigador aclarar las que quedaron confusas en un primer momento ${ }^{15}$.

Asegura Punch (2002: 323) que hay tres posibles explicaciones sobre por qué la investigación realizada con niños es, o debería ser, diferente de la realizada con adultos. Unos se fijan en la correspondencia existente entre los dos colectivos que prevalece en las relaciones sociales y asumen que existe una disimetría de poder que se produce por la situación de dependencia que los adultos reservan para los niños. Algo que, a la postre, fomenta que estos últimos asuman una posición de subordinación y que no estén acostumbrados a ser tratados como iguales por los primeros. Desde otra perspectiva, se entiende que los niños se encuentran en una fase evolutiva diferente a la de los adultos y que hay que adaptar la investigación a esas necesidades y carencias específicas de la infancia y la juventud. Un tercer planteamiento se centra en las limitaciones del investigador para acceder al conocimiento infantil y asume que es precisa una formación específica para validar la información ofrecida por los niños, que no puede ser interpretada desde una posición adultocéntrica.

También se podría decir que la forma de encarar una investigación (ética) tiene que ver con «las actitudes, los valores, las creencias y asunciones» en la forma de entender a los implicados, sea este el propio investigador, su equipo

13. De hecho, Morrow (2008: 56) destaca que la forma de solicitar la participación en la investigación condicionará el tipo de intervención del menor. Así, destaca que, por ejemplo, cuando se pregunta al grupo de clase estando todos presentes, lo más probable es que todos estén de acuerdo en participar, pero habrá unos cuantos que no intervendrán en las discusiones o no responderán a las preguntas.

14. Un ejemplo de utilización de la entrevista grupal para estudiar los «procesos de construcción del mundo social de la infancia» lo ofrece Rodríguez Pascual (2006).

15. Aseguran los autores que esa relación con el menor, aparte de mejorar su participación, favorece la fiabilidad de la información porque esta ha sido contrastada con él, así como su validez y su correcta interpretación (Thomas y O’Kane, 1998: 341-342). 
o los sujetos investigados (Graham et al., 2015: 332). El poder no es un hecho, sino que se demuestra con la propia acción (Gallagher y Gallagher, 2008). De manera muy oportuna, Rodríguez Pascual (2007: 89) asegura que «la propia investigación es una práctica cultural que revela mucho sobre nuestra manera de concebir la infancia o, lo que es lo mismo, nuestra representación social de la infancia». Por ello, para que el investigador quiera adaptar su investigación o integrar al niño en la misma, tiene que considerarle un sujeto con una opinión propia y con algo interesante que aportar, al mismo tiempo que tiene que situarse en una posición horizontal respecto al menor, a fin de que cada uno aporte algo importante para el desarrollo de la investigación. Ya avanzábamos que, desde la ratificación de la $\mathrm{CDN}$, las normas y las prácticas institucionales con niños y niñas, incluidas las relacionadas con la investigación, tienen que adaptarse para permitirles desarrollarse como actores sociales y participar en la vida social. Como aseguran Christensen y Prout (2002: 478), considerar a los menores como actores sociales nos coloca ante nuevos dilemas éticos y supone nuevas responsabilidades para el investigador.

Punch (2002: 322-323) pone en relación la forma de ver la infancia con el esfuerzo por adaptar o no la metodología de la investigación. Considera que quienes ven a los niños como maduros y competentes tenderán a utilizar los mismos métodos de investigación que con adultos. En ese caso, se augura un posible fracaso a la hora de gestionar el desequilibrio de poder, realmente existente, entre el investigador y el menor. Quienes tengan una visión del niño como de un ser totalmente diferente al adulto, tenderán a utilizar métodos etnográficos, que exigen una inversión prolongada en el contexto infantil, aunque no les permitan formar parte del mismo ${ }^{16}$. Por último, quienes consideran a los niños como adultos pero con diferentes competencias, tenderán a buscar técnicas de investigación innovadoras, que no siempre son bien utilizadas o comprendidas por los investigadores, dado que exigen una formación especializada para su utilización y, sobre todo, para la interpretación posterior de los resultados ${ }^{17}$.

Esta adaptación metodológica se relaciona con la entidad de la participación del menor. Así, French y Swai (1997, en Punch, 2002: 334) distinguen entre una investigación participativa, en la que están activamente implicados en la generación de información, y una investigación emancipatoria, en la que los participantes tienen el control sobre todo el proceso de investigación. Para fomentar una investigación participativa, el investigador tiene que adaptar los métodos a las necesidades especiales de los niños y de las niñas, siendo conscientes de que las diferencias atenderán a la situación concreta del menor,

16. Pereira y Barros (2011: 146) destacan que «los niños no están aislados en la sociedad en la que se integran, sino que interactúan con ella, produciendo cambios en todas las categorías que componen la estructura social, al mismo tiempo que componen su propia cultura y, consecuentemente, sus propios mundos sociales».

17. Para Kirk (2007: 1257), el problema es que a los niños no siempre les gusta dibujar, lo perciben como una forma de examen o no lo consideran interesante. Además, puede que el propio investigador no tenga la formación adecuada para interpretar y analizar los dibujos o que tienda a analizarlos con una visión adulta o, incluso, superficial. 
su forma de comunicación, sus necesidades específicas y el significado que atribuye a las palabras (Christensen y Prout, 2002: 483). Consideran que es obligación del investigador esforzarse porque el investigado tenga voz y se le escuche adecuadamente (al margen de su edad o madurez) $)^{18}$. Algo más complejo resulta fomentar una investigación emancipatoria e incorporar al niño al equipo y al proceso de investigación ${ }^{19}$. Quizás es posible hacerlo en la definición de temas de interés, en la realización de la propia investigación, en la interpretación de los resultados o en la difusión de la información una vez realizada. No obstante, parece más complejo, por ejemplo, integrar al menor en el proceso de análisis de datos cuantitativos, puesto que exige la utilización de herramientas que solo poseen los investigadores (Kirk, 2007: 1255).

Sobre esta cuestión de si debemos pensar en una metodología child-friendly, esto es, adaptada a la población infantil a la que se dirige, se ha generado una intensa bibliografía con más preguntas que respuestas. Una de las paradojas, que resume las demás, la plantea Punch (2002: 321) cuando percibe que, desde la sociología de la infancia, los mismos que claman por innovar y adaptar métodos de investigación son los que reivindican la competencia y la capacidad de los niños para participar en la investigación. La autora asegura que si los niños son considerados como actores sociales con capacidad para participar, quizás no sea preciso adaptar los métodos. Se puede afirmar que no existe tal contradicción, porque, para que los niños puedan ser realmente actores sociales y participar en las decisiones que les afectan y que les importan, sí que es preciso realizar una adaptación metodológica. A través de las distintas opciones metodológicas, se trata de colocar en una situación de igualdad a personas que no presentan coincidencias. Dado que la igualdad no está en el punto de partida, debe ser el resultado y la consecuencia de una adaptación metodológica.

\subsection{Participación del menor filtrada (por los porteros)}

Otra de las cuestiones que marca la diferencia entre la investigación con niños y con adultos es la necesaria existencia de porteros que limiten el acceso del investigador a los niños y niñas, anteponiendo generalmente su protección. Son ellos los que deciden si es posible, interesante o conveniente la investigación para quienes que se encuentran bajo su tutela o supervisión. De hecho, el consentimiento del menor del que hablábamos antes solo se produce cuando previamente ha habido un adulto que ha dado su aprobación. Y ello pese a que debería ser el consentimiento del menor el que «resultara fundamental para cualquier investigación» (Morrow, 2008: 54). Powell y Smith (2009: 136) consideran que esta «jerarquía de consentimientos» incrementa las potenciales

18. Aseguran que la mejor manera de investigar a niños no es hacerse pasar por niño, porque ellos no aceptan a los adultos como un miembro de su colectivo de edad (Kirk, 2007: 1255) o consideran que no son sinceros (Christensen, 2004: 174).

19. Sobre los riesgos y las posibilidades de que el menor sea el propio investigador, se puede consultar, como muestra, Gaitán y Liebel (2011: 171-182). 
barreras a la participación efectiva e igualitaria de los niños en la investigación. De hecho, la protección de los padres o cuidadores puede condicionar el acceso a los menores en función de los beneficios o perjuicios que, según ellos, pueda tener la participación infantil en la investigación. Por ello se ha insistido en la importancia de cuidar esta relación con los porteros sin intentar ser el experto, preguntando cuestiones que puedan resultar de su interés, devolviéndoles información que pueda ser utilizada en su práctica diaria y siendo conscientes de que atender al investigador le puede suponer un trabajo extra o que puede ver la investigación como una forma de fiscalización de su propio trabajo con los niños (Vaughn et al., 2012: 7-8).

En muchas ocasiones, los padres, los comités de ética, las organizaciones, los profesionales, los profesores o los cuidadores se presentan como barreras «protectoras» entre el investigador y el menor investigado, hablan supuestamente por boca de los niños alegando que estos tienen muchas otras tareas (más importantes) que hacer en ese momento preciso, que no van a entender el sentido de la investigación o las preguntas que se formulan, que no van a estar interesados (Thomas y O'Kane, 1998: 346; Powell y Smith, 2009: 126; Hood et al., 1996) o que sus planteamientos, directamente, no son interesantes. En otras, un exceso de celo protector llega a impedir la participación del menor en una investigación que puede resultar de su interés individual o colectivo (Kirk, 2007: 1253), o que versa sobre un tema que entienden que puede ser doloroso o traumático para el menor, sin haber discutido con él sobre este aspecto. Como indican Powell y Smith (2009: 128), la concepción que se tiene de la infancia como incondicionalmente vulnerable hace que se entienda que casi cualquier tema puede ser doloroso o traumático para un niño ${ }^{20}$, algo que puede condicionar todo el posterior proceso de filtro por los comités éticos, de selección de la población objeto de la investigación y de definición de los métodos de investigación.

En definitiva, Graham et al. (2015: 332) destacan que, en el centro de las discusiones éticas en la investigación con niños, parece existir una tensión entre «la necesidad de protección y la exigencia de participación». Aseguran los autores citados que, en esa tensión, parece imponerse la protección sin considerar que «la no participación puede ser más perjudicial que la participación, porque se les niega a los niños las ventajas asociadas a la participación, incluyendo la educación, la terapia, el empoderamiento, junto con la posibilidad de acceder a recursos». Además, esa escisión entre protección y participación desconoce la indivisibilidad de los derechos que plantea que «la verdadera protección de los niños exige que todos los derechos, incluidos los derechos de participación, se respeten» Graham et al. (2015: 332). Así, como reconocía Sandbaek (1999, en Powell y Smith, 2012: 139), "la discusión ética en la investigación puede reducirse a un conflicto de derechos, el derecho del niño a ser protegido versus

20. Powell y Smith (2012: 126) reconocen que a algunos niños tutelados por el Estado se les deniega la oportunidad de participar en la investigación porque se presupone su vulnerabilidad y se entiende que es preciso protegerles. 
su derecho a opinar». Conflicto que puede convertirse, finalmente, en una situación de exclusión (Powell y Smith, 2012: 133).

\section{Conclusiones: sobre las consideraciones éticas y el respeto de los derechos de las personas investigadas}

Me parece oportuno concluir con algunas consideraciones sobre la relación entre la reflexión ética y el proceso de investigación que se pueden trasladar a la investigación con niños y niñas por ser importantes en la realización de sus derechos. En primer lugar, con carácter general, se podría avanzar que la mayor o menor consideración sobre las cuestiones éticas en la investigación se relacionan con el modo que tiene el investigador de entender el sentido y la finalidad de su propio trabajo. Así, dependerá de si considera que los potenciales resultados de la investigación son un objetivo incondicional a lograr o si asume que no todo vale y no se puede hacer investigación a cualquier precio y pasando por encima del respeto hacia los derechos de las personas investigadas y de los principios éticos considerados esenciales por la comunidad científica ${ }^{21}$, y más aún cuando las personas investigadas son niños a los que tenemos la obligación de proteger y también de dispensarles un trato digno. Si no tiene claros estos presupuestos, tenderá a ver las cuestiones éticas de la investigación o las relacionadas con los sujetos investigados como trámites a cumplimentar o, en el peor de los casos, como "obstáculos» a sortear para lograr el objetivo último, que es el de realizar una investigación con unos supuestos efectos sociales o reconocimientos académicos.

En segundo lugar, la investigación debe comportar un proceso de evaluación constante de las consideraciones éticas en el proceso de investigación, de sus efectos y resultados (Bell, 2008: 8). Algo que, para Harcourt y Quennerstedt (2014: 3), no solo supone una responsabilidad «institucional» del investigador hacia los comités de ética encargados de verificar los preliminares y el planteamiento de la investigación, o «hacia la comunidad científica para producir investigación de calidad», sino que también (yo diría principalmente) debe traducirse en una actitud problematizadora y atenta a los posibles dilemas éticos que se produzcan a lo largo de toda la investigación y que pongan en jaque el respeto hacia las personas investigadas y sus derechos. Se trata de una ética práctica o de una ética en la práctica que exige al investigador y a su equipo una constante evaluación crítica, entre sí y con los sujetos investigados, del proceso investigador que le permita adelantarse a los posibles desafíos que se van planteando.

En tercer lugar, por las implicaciones en el respeto de los derechos, entiendo que la consideración de cuestiones éticas debe ser una condición sine qua non de la investigación. Esto es, una investigación que desconozca las

21. Bell (2008: 14) hace referencia a la reticencia de las Social Research Association Ethical Guidelines, de 2003, que indican que el respeto de derechos y libertades solo se refiere a las instituciones públicas, no cuando la investigación se realiza por parte de instituciones privadas. 
cuestiones éticas mínimas y no respete la dignidad de las personas implicadas en ella, al margen de su edad o su capacidad para comprender, no puede valer como tal. No vale, ni está justificada la investigación a cualquier precio. Sobre todo porque ese precio es considerar a las personas investigadas como simples objetos de examen, útiles en la obtención de información. Se trata de poner en valor cuestiones básicas que, según la International Charter for Ethical Research Involving Children, tienen que ver con una investigación que garantice la autonomía de los participantes para involucrarse en ella o para abstenerse de hacerla, prevenga o minimice cualquier riesgo que pudiera derivarse de su realización o que fomente que todos los participantes sean tratados de forma justa. Algo que debe traducirse en una actitud constante del investigador que proteja los derechos de las personas implicadas en la investigación y sea consciente de que la participación en ella puede ser intrusiva y causar daños ${ }^{22}$. Cuestiones básicas que, como Harcourt y Quennerstedt (2014: 3) destacan, tienen que ver con una investigación realizada con mérito e integridad, con el hecho de que sea justa (en la selección de los sujetos, en el reparto de riesgos y daños), que los potenciales daños estén justificados en los beneficios a lograr, que se trate de una investigación que respete el bienestar, la seguridad de las personas, su cultura y sus creencias, su intimidad y sus habilidades.

En esa línea, Christensen y Prout (2002: 490) recuerdan algunas de las cuestiones a considerar por el equipo de investigación en todo estudio con niños y jóvenes que se pretenda ético y respetuoso de sus derechos. Así, insisten en que el objeto de la investigación debe suponer algún beneficio para la infancia; debe lograr un equilibrio entre costes y beneficios para el menor (tiempo de dedicación; intrusión en su vida; ansiedad generada por el tema, el proceso o el método de investigación; miedo por las consecuencias de participar en ella o no; coerción para participar en ella o no; etc.); debe respetar la privacidad y la confidencialidad, que deben ser verificadas antes de que se use la información; habrá que revisar los métodos de selección y participación de los menores ${ }^{23}$; verificar los mecanismos y las instituciones de financiación y sus consecuencias; establecer mecanismos de revisión de la investigación y sus resultados; definir qué información se tiene que ofrecer a niños y padres o profesores para lograr el consentimiento; verificar que el consentimiento es libre y sin consecuencias; ofrecer información (o vías para obtenerla) sobre los resultados de la investigación a niños, padres o profesores, y analizar qué implicaciones tendrá para el grupo de niños. En todo caso, es preciso ser consciente de que un trabajo de estas características que se pretenda ético y que sea practicado con jóvenes y adolescentes supone una mayor inversión de tiempo y una consideración

22. Sobre todo porque hay que «tomar en consideración los posibles efectos dañinos de "tener que hablar"» (Komulainen, 2007: 25).

23. Teniendo en cuenta que, como indican Beazley et al. (2004: 374), los procesos de selección e identificación de los participantes también pueden ser estigmatizantes; por ejemplo: cuando se les selecciona porque son niños de la calle, con problemas de drogadicción, delincuencia, violentos, etc. Aseguran que, cuando fracasamos a la hora de interpretar la propia percepción del niño, violamos su dignidad. 
no lineal del proceso seguido. Se trata de un sacrificio que, quizás, no todos los investigadores están dispuestos a hacer, pero que la comunidad científica y social debe exigir.

En definitiva, todas esas consideraciones éticas y metodológicas en la investigación social con niños son necesarias por varias razones. Una, por razones normativas, para evitar que el derecho del niño a participar en los estudios sobre temas que le incumban sea ajeno a su vulnerabilidad y le suponga más daño que beneficio. Dos, por razones pragmáticas para la propia investigación, para conseguir una participación más significativa y un conocimiento más auténtico de las opiniones de niños y adolescentes. Tres, por razones sociales, porque la voz de los niños adecuadamente recogida (con métodos adaptados a sus capacidades comunicativas) y analizada (evitando interpretaciones adultocéntricas) permite una mejor comprensión de la realidad social por parte de los menores y una evolución en los procesos de implementación de sus derechos. Finalmente, las cuestiones éticas deben integrarse en las de tipo metodológico, porque si los errores metodológicos invalidan los resultados de la investigación, los de tipo ético la deslegitiman ante la comunidad científica.

\section{Referencias bibliográficas}

Beazley, H.; Bessell, Sh.; EnNew, J. y Waterson, R. (2009). «The right to be properly researched: research with children in a messy, real world». Children's Geographies, 7 (4), 365-378. <https://doi.org/10.1080/14733280903234428>

BeLL, N. (2008). «Ethics in child research: Rights, reason and responsibilities». Children's Geographies, 6 (1), 7-20. <https://doi.org/10.1080/14733280701791827>

Bernuz Beneitez, M.J.; Fernández Molina, E.; Gómez, D. y Vicente, C. (en prensa). La ética en la investigación criminológica.

Chakraborty, K.; NAnSE, B.; GibBS, L. y McDougall, C. (2012). «Ethical negotiations: Committees, methods and research with children». International Journal of Children's Rights, 20, 541-553. <https://doi.org/10.1163/157181812x634472>

CHRISTENSEN, P. (2004). «Children's participation in ethnographic research: Issues of power and representation». Children and Society, 18, 165-176. <https://doi.org/10.1002/chi.823>

Christensen, P. y Prout, A. (2002). «Working with ethical symmetry in social research with children». Childhood, 9 (4), 477-497. <https://doi.org/10.1177/0907568202009004007>

FERNANDES, N. (2016). «Ética na pesquisa com crianças: Ausências e desafíos». Revista Brasileira de Educaçao, 21 (66), 759-779. <https://doi.org/10.1590/s1413-24782016216639>

Gaitán Muñoz, L. (2006). Sociología de la infancia. Madrid: Síntesis.

- (2006). «La nueva sociología de la infancia: Aportaciones de una mirada distinta». Politica y Sociedad, 43 (1), 9-26.

GatTÁn Muñoz, L. y Liebel, M. (2011). Ciudadania y derechos de participación de los niños. Madrid: Síntesis. 
Gallagher, L.A. y Gallagher, M. (2008). «Methodological immaturity in childhood research?: Thinking through "participatory methods"». Childhood, 15 (4), 499-516. <https://doi.org/10.1177/0907568208091672>

GraHAM, A.; POWELl, A. y TAYLOR, N. (2015). «Ethical research involving children: Encouraging reflexive engagement in research with children and young people». Children and Society, 29, 331-343. <https://doi.org/10.1111/chso.12089>

Graham, A.; Powell, M.; Taylor, N.; Anderson, D. y Fitzgerald, R. (2013). Investigación ética con niños. Florencia: Centro de Investigaciones de UNICEF / Innocenti.

Harcourt, D. y Quennerstedt, A. (2014). «Ethical guardrails when children participate in research: Risk and practice in Sweden and Australia». Sage Open, july-september, 1-8. <https://doi.org/10.1177/2158244014543782>

Hood, S.; Kelley, P. y Mayall, B. (1996). «Children as Research Subjects: A Risky Enterprise». Children and Society, 10, 117-128. <https://doi.org/10.1111/j.1099-0860.1996.tb00462.x>

IgNATIEFF, M. (2000). The rights revolution. Toronto: House of Anantis Press Limited.

James, A. (2007). "Giving voice to children's voices: Practices and problems, pitfalls and potentials». American Anthropologist, 109 (2), 261-272. <https://doi.org/10.1525/aa.2007.109.2.261>

KIRK, S. (2007). «Methodological and ethical issues in conducting qualitative research with children and young people: A literature review». International Journal of Nursing Studies, 44, 1250-1260. <https://doi.org/10.1016/j.ijnurstu.2006.08.015>

Komulainen, S. (2007). "The ambiguity of the child's "voice" in social research". Childhood, 14 (1), 11-28. <https://doi.org/10.1177/0907568207068561>

Morrow, V. (2008). «Ethical dilemmas in research with children and young people about their social environments». Children's Geographies, 6 (1), 49-61. $<$ https://doi.org/10.1080/14733280701791918>

Pereira, B.E. y Barros Pedroso, N. (2011). «De objetos a sujeitos de pesquisa: Contribuiçoes da sociologia da infância au desenvolvimento de uma etnografia da educaçao de crianças de populaçoes tradicionais». Educaçao: Teoria e pratica, 21 (36), 138-156.

Powell, M.A. y SMiTh, A.B. (2009). "Children's participation rights in research». Childhood, 16 (1), 124-142. <https://doi.org/10.1177/0907568208101694>

PunCH, S. (2002). «Research with children: The same or different from research with adults?». Childhood, 9 (3), 321-341. <https://doi.org/10.1177/0907568202009003005>

Richards, S.; Clark, J. y BOGGIS, A. (2015). Ethical Research with Children: Untold narratives and taboos. Londres: Palgrave McMillan.

Rodríguez Pascual, I. (2006). «Redefiniendo el trabajo metodológico cualitativo con niños: El uso de la entrevista de grupo aplicado al estudio de la tecnología». Revista de Metodología de Ciencias Sociales, 12, 65-88. <https://doi.org/10.5944/empiria.12.2006.1135>

- (2007). Para una sociología de la infancia: Aspectos teóricos y metodológicos. Madrid: CIS. 
Spyrou, S. (2011). «The limits of children's voices: From authenticity to critical, reflexive representation». Childhood, 18 (2), 151-165. <https://doi.org/10.1177/0907568210387834>

Thomas, N. y O'Kane, C. (1998). "The ethics of participatory research with children». Children and Society, 12, 336-348. <https://doi.org/10.1111/j.1099-0860.1998.tb00090.x>

Vaughn, M.G.; PetTus-Davis, C. y ShOOK, J.J. (2012). Conducting research in Juvenile and Criminal Justice Settings. Oxford: Oxford University Press.

Willumsen, E.; HugaAs, J.V. y StudsRod, I. (2014). "The child as co-researcher: Moral and Epistemological issues in childhood research». Ethics and Social Welfare, 8 (4), 332-349.

<https://doi.org/10.1080/17496535.2014.894108> 